\title{
A high-efficiency and compact charge pump with charge recycling scheme and finger boost capacitor
}

\author{
Hui Peng*, Pieter Bauwens, Herbert De Pauw, and Jan Doutreloigne \\ CMST, Gent University-IMEC, Technologiepark 15 B-9052 Gent, Belgium
}

\begin{abstract}
A 16-phase 8-branch charge pump with finger boost capacitor is proposed to increase the power efficiency. Compared with the standard capacitor, the finger capacitor can significantly reduce the parasitic capacitance. The proposed four-stage charge pump with finger capacitor can achieve 14.2 V output voltage from a $3 \mathrm{~V}$ power supply. The finger capacitor can increase the power efficiency of the charge pump to $60.5 \%$ and save chip area as well.
\end{abstract}

\section{Introduction}

In the last decade the wearable and portable electronic device market has shown an explosive increase. The electronic device size and the battery autonomy are two important evaluation factors for consumers. In the electronic devices the DC-DC converter is a necessary module to bridge the voltage difference between the battery supply and the requirements of other functional modules. The capacitive DC-DC converter and the inductor-based DC-DC boost converter are two popular topologies to step up the DC voltages. The inductorbased DC-DC converter normally has relatively high efficiency and can supply more power, but it also needs a bulky external inductor which needs more space and is not suitable for the tightly compact devices. In contrast, the capacitive DC-DC converter has the advantage that it can be integrated in monolithic chips and significantly save space. The Dickson charge pump (CP) is an extensively used capacitive converter [1] and a 4-stage Dickson CP is shown in Fig. 1. Compared with the inductor-based converter, a Dickson CP always experiences relatively low efficiency which is attributed to the parasitic capacitance $\left(\mathrm{C}_{\mathrm{P}}\right)$ of the flying boost capacitor (C). In each clock period, the parasitic capacitance always needs to be charged and discharged, thereby causing power losses, but it doesn't make any useful contribution to the $\mathrm{CP}$ operation. As the energy stored in the battery is limited, it is necessary to design a

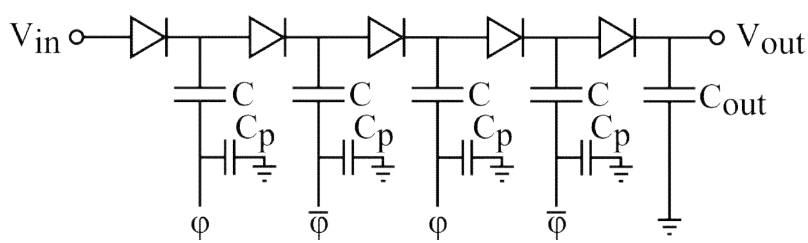

Fig. 14 -stage Dickson charge pump

\footnotetext{
* Corresponding author: Hui.Peng@UGent.be
}

$\mathrm{CP}$ with high efficiency and low power consumption in order to extend the electronic device's battery autonomy.

One way to minimize the effect of parasitic capacitance is the charge recycling method. This method can save $50 \%$ of the power consumption attributed to the parasitic capacitance. In our previous work [2], a 16phase 8-branch CP is introduced which is also based on the charge recycling concept but has an advanced charge recycling strategy. This new $\mathrm{CP}$ has 7 intermediate voltage levels during the charge recycling process which can save $87 \%$ of the power consumption related to the parasitic capacitance. Apart from the charge recycling method, another straightforward way to boost the power efficiency is by decreasing the parasitic capacitance of the boost capacitor itself. In this work we use the top metal layers to design several finger structure capacitors. The ratio of parasitic capacitance to the nominal capacitance of finger capacitors with different configurations is investigated and compared with standard capacitors in the CMOS technology library. Finally the finger capacitor is used in the 16-phase 8branch CP which can significantly increase the power efficiency compared with the $\mathrm{CP}$ using the standard capacitors.

\section{Circuit Implementation}

\subsection{6-phase 8-branch CP}

Charge recycling is a useful method to improve the power efficiency and it has been demonstrated in several papers $[3,4]$. In our previous work the concept of charge recycling was extended and implemented in a 16-phase 8-branch CP, yielding an improved power efficiency as reported in [2]. Fig. 2 (a) shows one stage of the 16phase 8-branch $\mathrm{CP}$, where the bottom plates of the boost capacitors $\mathrm{C}_{1}-\mathrm{C}_{8}$ are connected by a transmission gate (TG) matrix (as shown in Fig. 2 (b)) to implement the 


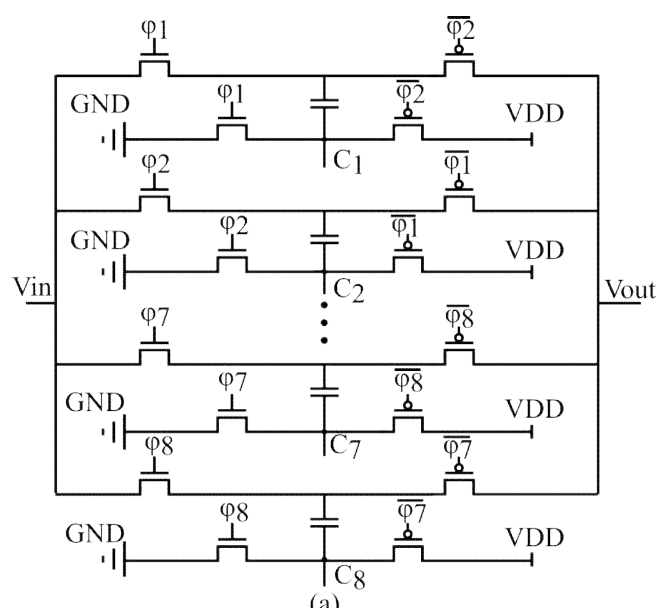

(a)

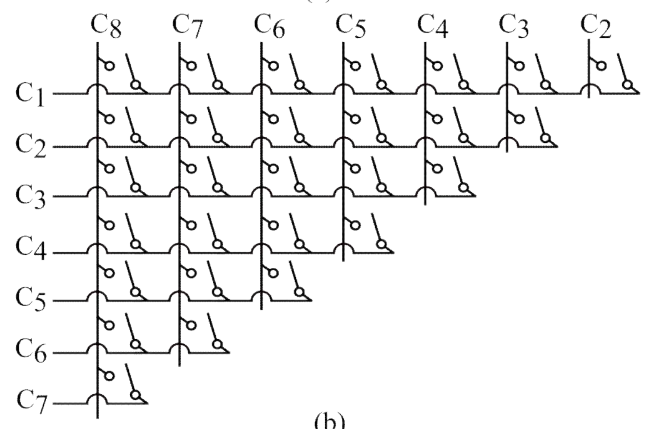

(b)

Fig. 2 (a) One stage of the 16-phase 8-branch CP structure (b) TG matrix

charge recycling strategy. Fig. 3 shows the waveform of the boost capacitor's bottom plate $\mathrm{C}_{1}$ in one stage and the charge recycling procedure. The principle of charge recycling in this 16-phase 8-branch $\mathrm{CP}$ is that instead of charging the bottom plate of boost capacitor $\mathrm{C}_{1}$ directly from GND to VDD, it will first be properly shorted by the transmission gate matrix to the bottom plate of other boost capacitors, in such a way that a 9-level triangular waveform is obtained. This strategy can significantly decrease the power dissipation due to parasitic capacitance and hence increase the power efficiency, as well as decrease the output voltage ripple.

\subsection{The structure of the finger capacitors}

In the monolithic CP design, a big challenge is that the

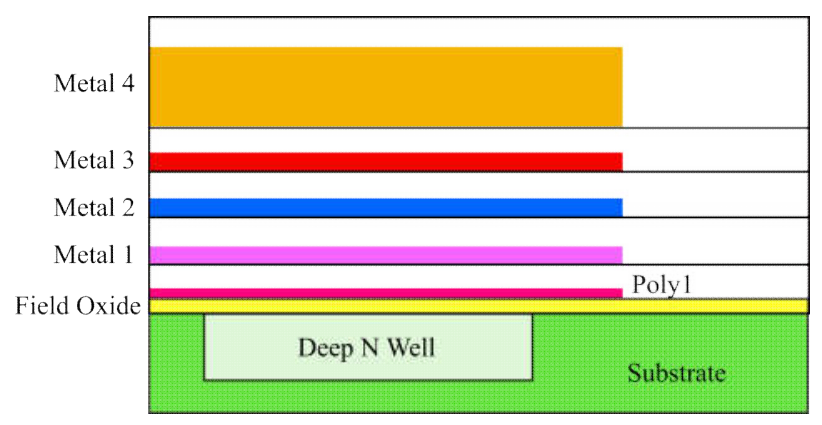

Fig. 4 Cross-sectional view of all the used layers in H35 CMOS technology

expected output voltage of the $\mathrm{CP}$ is normally higher than the voltage tolerance of a standard CMOS technology. Therefore a high-voltage (HV) CMOS technology should be used in the CP design. The 0.35 $\mu \mathrm{m}$ H35 CMOS IC technology of Austria MicroSystems (AMS) is a typical high-voltage CMOS technology and the cross-sectional view of the relevant layers in this technology is shown in Fig. 4. There are three standard types of capacitors in this technology and they are the CPOLY (Poly 1 - Poly 2), CPM (Poly 1- Metal 1 - Metal 2 - Metal 3) and CWPM (Deep N Well - Poly 1 - Metal 1 - Metal 2 - Metal 3) capacitors respectively. The CPOLY capacitor has the highest nominal capacitance but its maximum operation voltage is only $5.5 \mathrm{~V}$, which is too low to work as boost capacitor. For the CPM and CWPM capacitors, their maximum operation voltages are $120 \mathrm{~V}$ and $70 \mathrm{~V}$ respectively, which is high enough for most portable electronic devices. But these two types of capacitors both experience a high ratio of parasitic capacitance to nominal capacitance. For a CPM capacitor with an area of $115 \mu \mathrm{m} * 115 \mu \mathrm{m}$ for example, the parasitic capacitance and nominal capacitance are $1.597 \mathrm{pF}$ and $1.7 \mathrm{pF}$ respectively, the ratio being as high as $94 \%$. One way to solve this problem is that we only use the several metal layers on the top side to design the capacitor, so the parasitic capacitance can be significantly reduced. If we use the layers Metal 2 till Metal 4 to design a $115 \mu \mathrm{m} * 115 \mu \mathrm{m}$ flat metal capacitor (CM), Metal 2 and Metal 4 being

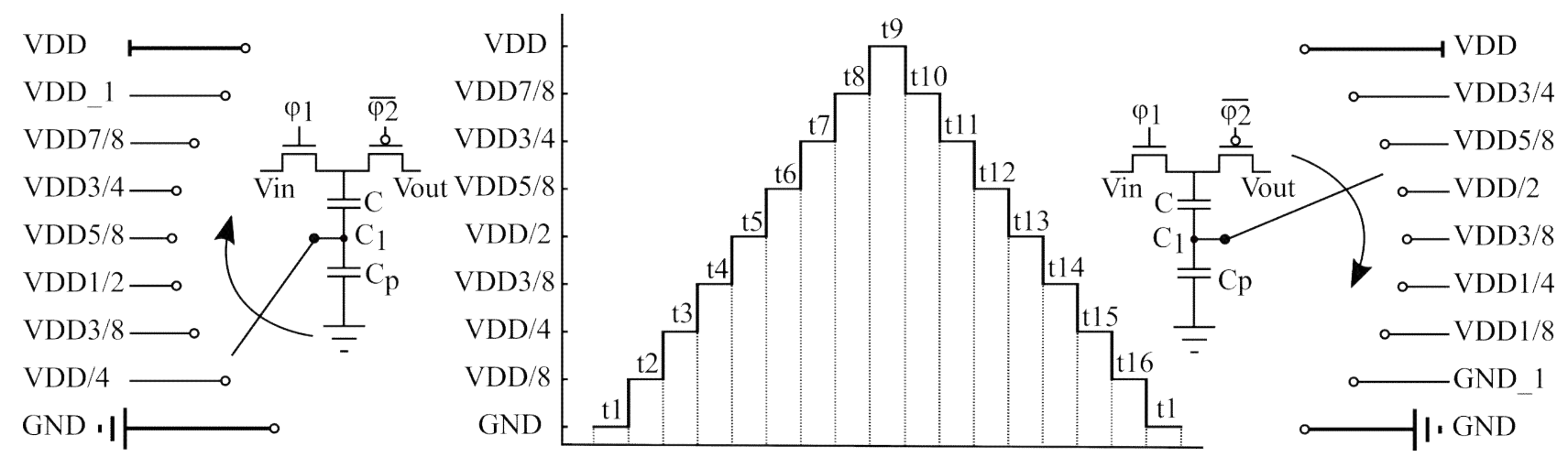

Fig. 3 16-phase 8-branch CP boost capacitor's bottom plate waveform 

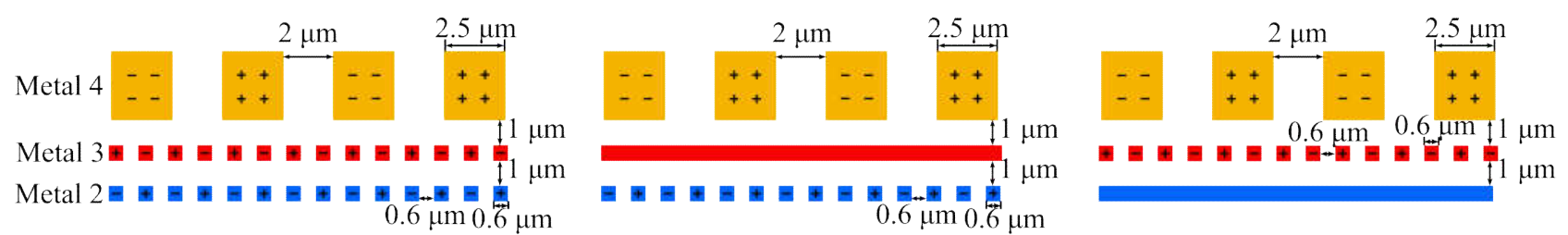

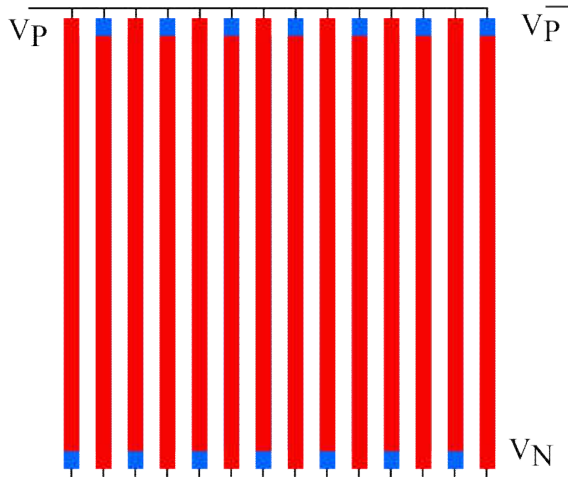

(a)

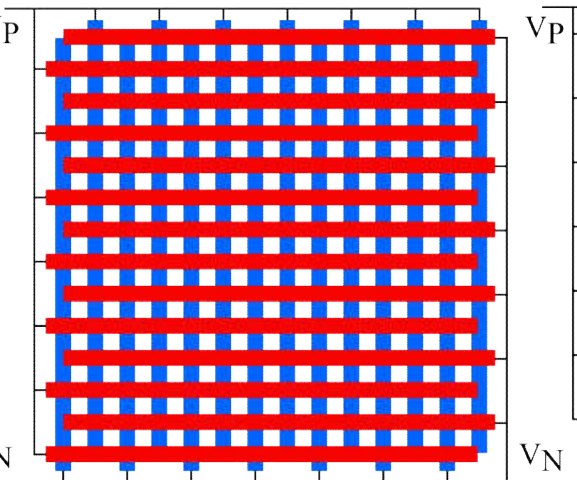

(b)

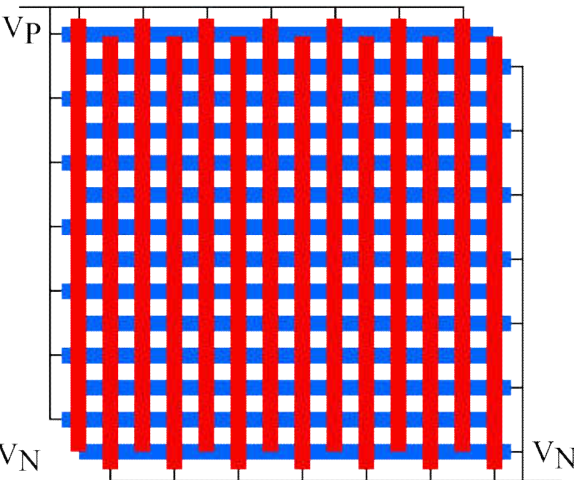

(c)

Fig. 5 The finger capacitors with different configurations

Table 1. The capacitance of different capacitor types $(115 \mu \mathrm{m}$

\begin{tabular}{|c|c|c|c|}
\hline Capacitor & $\begin{array}{c}\text { Nominal } \\
\text { Capacitance } \\
\text { [pF] }\end{array}$ & $\begin{array}{c}* 115 \mu \mathrm{m}) \\
\text { Parasitic } \\
\text { Capacitance } \\
{[\mathbf{p F}]}\end{array}$ & $\begin{array}{c}\text { Ratio of Parasitic } \\
\text { to Nominal } \\
\text { Capacitance [\%] }\end{array}$ \\
\hline CPOLY & 11.4 & 1.588 & 13.9 \\
\hline CPM & 1.7 & 1.597 & 94.0 \\
\hline CWPM & 3.3 & N/A & N/A \\
\hline CM & 0.979 & 0.185 & 18.9 \\
\hline CFM_a & 1.962 & 0.093 & 4.74 \\
\hline CFM_b & 1.887 & 0.093 & 4.93 \\
\hline CFM_c & 1.893 & 0.093 & 4.91 \\
\hline
\end{tabular}

interconnected as one plate of the capacitor and Metal 3 serving as the other plate, the ratio of parasitic to nominal capacitance is reduced to $18.9 \%$. However, the nominal capacitance is only $0.979 \mathrm{pF}$, as shown in Table 1. In order to achieve at the same time the highest possible nominal capacitance, we designed the finger structure capacitors (CFM) by using the Metal 2 to Metal 4 layers with different configurations. The CFM capacitors' structures are shown in Fig. 5 and the corresponding nominal and parasitic capacitance values are shown in Table 1. The CFM capacitors have a reasonable nominal capacitance and very little parasitic capacitance, with the CFM_a capacitor having the lowest ratio of parasitic to nominal capacitance of only $4.74 \%$ between one plate and the substrate.

\subsection{Control circuit}

Fig. 6 shows the on-chip control circuit of this 16-phase 8-branch CP. The frequency of this control circuit is determined by the voltage $\mathrm{V}_{\mathrm{C}}$ which is connected to the gate of p-type MOSFET $\mathrm{P}_{1} . \mathrm{V}_{\mathrm{C}}$ controls the drain current of $\mathrm{P}_{1}$, which drives the unity-gain current mirror $\mathrm{N}_{1}+\mathrm{N}_{2}$, resulting in a ring oscillator bias current equal to the current through $\mathrm{P}_{1}$. The ring oscillator is comprised of 17

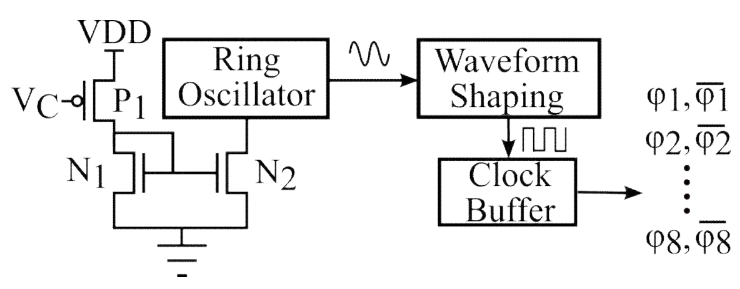

Fig. 6 The schematic of the control circuit

inverters and the frequency can sweep from 0 to 9.32 $\mathrm{MHz}$ by varying the control voltage $\mathrm{V}_{\mathrm{C}}$. The signal from the oscillator then passes through the waveform shaping and clock buffer circuits to become the expected clock signals $\varphi_{1}, \overline{\varphi_{1}}, \varphi_{2}, \overline{\varphi_{2}} \ldots . \varphi_{8}, \overline{\varphi_{8}}$ to control the 16phase 8-branch CP.

\section{Simulation Results}

The performance of a 16-phase 8-branch CP with CFM_a, CM or CPM boost capacitors is simulated by using a $0.35 \mu \mathrm{m}$ CMOS technology. These three types of capacitors exhibit a ratio of parasitic to nominal capacitance of $4.74 \%, 18.9 \%$ and $94 \%$ respectively, as already stated in Table 1 . The layout of these three capacitors is shown in Fig. 7. They are all square and their side lengths are $116 \mu \mathrm{m}, 165 \mu \mathrm{m}$ and $125 \mu \mathrm{m}$ respectively in order to obtain the same nominal capacitance of $2 \mathrm{pF}$ for all boost capacitor types, which is essential to allow a fair comparison of the CP performance. The supply voltage VDD is $3 \mathrm{~V}$ and the clock frequency is set to $4 \mathrm{MHz}$. All the CPs have four stages which are connected in series. The first stage's node Vin is connected to VDD and a $20 \mathrm{pF}$ load capacitor is attached to the output node of the fourth stage. 


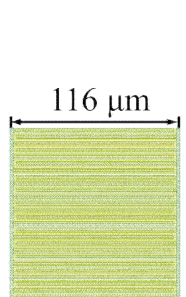

(a)

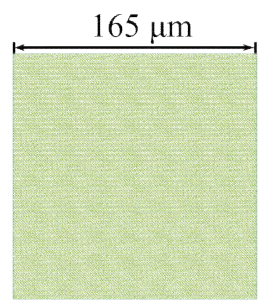

(b)

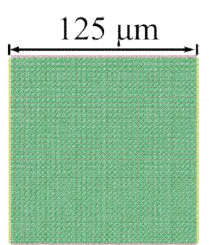

(c)
Fig. 7 The layout of (a) CFM_a, (b) CM and (c) CPM capacitors

The output voltage of the three CP circuits with different boost capacitors at different output currents is plotted in Fig. 8. For all these three CPs, the output voltage decreases equally fast when the output current increases. At zero-load condition, the CPs with CM and CFM_a capacitors have a similar output voltage of 14.2 $\mathrm{V}$, while the output voltage for the $\mathrm{CP}$ with $\mathrm{CPM}$ capacitor is a little lower, about $13.8 \mathrm{~V}$.

Fig. 9 shows the power efficiency of the three CP circuits with different boost capacitors at different output current. In the efficiency calculation, the power dissipation in the control circuit and all CP transistors is taken into account. The maximum efficiency of the CP with CPM boost capacitor is only $36.2 \%$ at $70 \mu \mathrm{A}$ current load. In contrast, at the same current load the power efficiency of the CP with CFM_a and CM capacitors is $57.2 \%$ and $55.4 \%$ respectively. The maximum efficiency of the CP with CFM_a and CM

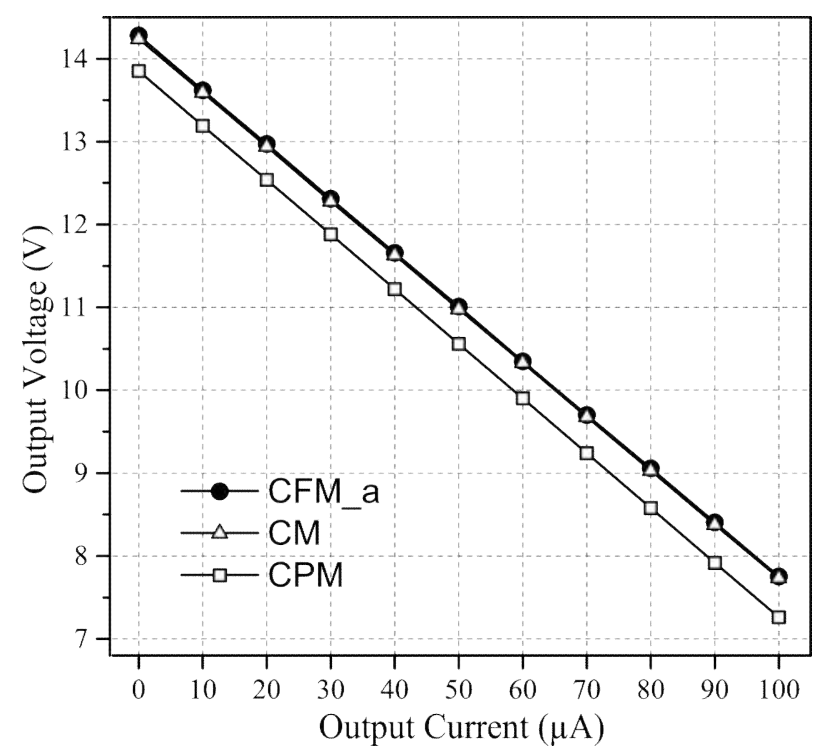

Fig. 8 Output voltage at different current load

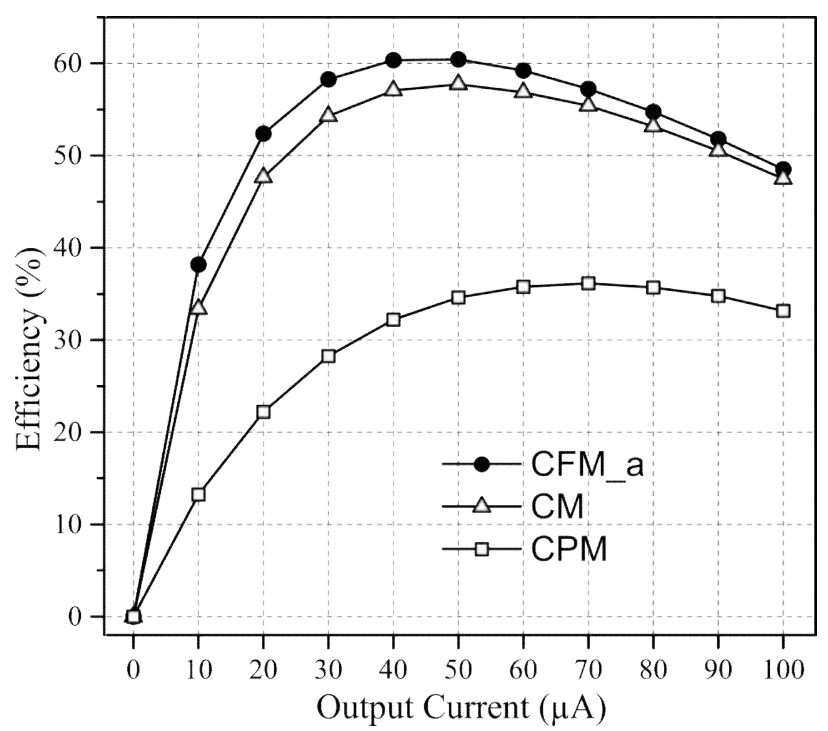

Fig. 9 Efficiency at different current load

capacitors is $60.5 \%$ and $57.8 \%$ respectively. It is worth mentioning that the CP with CFM_a boost capacitor not only has the maximum efficiency but also has the minimum chip area because for the same nominal capacitance of $2 \mathrm{pF}$ the CFM_a capacitor has the minimum size of $116 \mu \mathrm{m} * 116 \mu \mathrm{m}$.

\section{Conclusion}

In this work a 16-phase 8-branch $\mathrm{CP}$ with finger boost capacitor is proposed. By using the finger capacitor, the ratio of parasitic capacitance to nominal capacitance of the boost capacitor can be significantly reduced to only $4.74 \%$, compared to $94 \%$ for a standard poly-metal sandwich capacitor. By combining the charge recycling method and the finger capacitor, the output voltage of the proposed four-stage CP can reach $14.2 \mathrm{~V}$ from a $3 \mathrm{~V}$ power supply and the maximum efficiency is $60.5 \%$.

This work was supported by China Scholarship Council.

\section{References}

[1] J. F. Dickson, IEEE J. Solid-State Circuits, 11, 374-378 (1976)

[2] H. Peng, P. Bauwens, H. De Pauw, and J. Doutreloigne, EDSSC (2019)

[3] C. Lauterbach, W. Weber, and D. Romer, IEEE J. SolidState Circuits, 35, 719-723 (2000)

[4] J. Doutreloigne, WCECS, 811-817 (2010) 